\title{
A Proposed Turbo Coded Wavelet Packet Modulation based MC-CDMA
}

\author{
Aqiel Niama Almaamory \\ Assistant lecturer \\ Baghdad University $\backslash$ Electronic and Communications Engineering
}

\begin{abstract}
A turbo coded Discrete Wavelet Packet Transform DWPT based Multicarrier CDMA system has been proposed to enhance the bit error rate and complexity of the conventional MC-CDMA which based on Fast Fourier Transform FFT over different channel models. The decoding algorithm used max$\log$-MAP algorithm. The computer simulation tests show that the BER of the coded DWPT based MC-CDMA has been improved by $4 \mathrm{~dB}$ compared with the coded FFT based MCCDMA over Additive White Gaussian Noise AWGN and 4.5 $\mathrm{dB}$ over the multipath fading channel at of $\mathrm{BER}=10^{-3}$.
\end{abstract} Keywords: MPM MC CDMA, PCCC, MCM, Mallat's Algorithm, OFDM.

\section{General Terms}

MC-CDMA, Wavelet Transform, Turbo Coding

\section{INTRODUCTION}

Recently, the MC_CDMA technique based on the combination of conventional CDMA and OFDM technique has attracted great interest in both practical and theoretical studies of modern mobile communication because of its high spectral efficiency and high data transmission[1].

This combination is a useful technique for high speed systems which has the property of variable data rates as well as provides reliable communication system [2].

By using multi_carrier modulation, CDMA signal is spreaded over several carriers by which frequency diversity is achieved similar to path diversity in Rake receiver, but with lower complexity of equalization in the frequency domain [1].

In OFDM based wavelet transform, the IFFT and FFT blocks are simply replaced by an Inverse Discrete Wavelet Transform IDWPT and Discrete Wavelet Transform DWPT respectively[1].

The conventional MC_CDMA is implemented by means of FFT operation. In its frequency spectrum, the main lobe does not concentrate energy effectively and side lobe attenuates slowly, the multi path fading and synchronization error will cause severe performance degradation due to inter channel interference ICI, intersymbol interference ISI and multi-access interference MAI. Moreover, the FFT MC CDMA often restores to cyclic prefix $\mathrm{CP}$ to eliminate the ISI and maintain orthogonality between neighboring sub_carriers, which decreases the efficiency of spectrum utilization considerably in some communication scenarios.

Due to the higher spectral containment between sub channels, wavelet based OFDM can be better in combating narrowband interference and its inherently more robust with respect to ICI than traditional Fourier filter.
Without the $\mathrm{CP}$, the data rate in wavelet systems can surpass those of Fourier implementations, one of its key motivating factors.

So, the main and important difference between FFT and DWT based OFDM is that the wavelet based OFDM will not add a cyclic prefix to OFDM system [2]. This property makes the wavelet to combat with FFT for future system.

One method suggested to improve the performance of a communication system is to use the channel coding. Channel coding refers to the class of signal transformations designed to improve communication performance by enabling the transmitted signals to better withstand the effects of various channel impairments, such as noise, interference, and fading. In this work, a parallel concatenated convolutional code of two convolutional codes are used as a channel coding scheme to be used with the wavelet based MC-CDMA system. They showed through simulation that turbo codes are capable of achieving BER of the order of $10^{-5}$ at $\mathrm{E}_{\mathrm{b}} / \mathrm{n}_{\mathrm{o}}$ of just $0.7 \mathrm{~dB}$ of from the Shanon limit.

\section{WAVELET PACKET MCM}

A wavelet is a waveform of effectively limited duration that has an average value of zero. The comparative difference between wavelets and sine waves, which are the basis of Fourier analysis is that sinusoids do not have limited duration, they extend from minus to plus infinity and wheresinusoids are smooth and predictable, wavelets tend to be irregular and asymmetric[3].

Wavelet packet modulation is a multicarrier Communication technique where independent information carrying symbols are placed on orthogonal wavelet packet bases. The basic functions in wavelet packet representation are obtained from a single function called the motherwavelet through scaling and translations. Unlike OFDM which divides the whole bandwidth into orthogonal and overlapping subbands of equal bandwidths, OWDM using the discrete wavelet packet transform is a multiplexing transmission method in which data is assigned to wavelet sub bands having different time and frequency resolutions.

A weakness of DFT-based OFDM is the large spectral overlap between frequency responses of filters corresponding to different subchannels. This can lead to substantial leakage of power between subchannels and consequently induce inter channel interference. [4],[5].

\section{TURBO CODE (PARALLEL CONCATENATED CONVOLUTIONAL ECODING)}

The advantage of wavelet transform overother transforms such as Fourier transform is that it is discrete both in time as well as scale. One of the advantages of using wavelet transform is that 
due to the overlapping nature of wavelet properties, the wavelet based OFDM does not need cyclic prefix to deal with delay spreads of the channel. As a result, it has higher spectral containment than that of Fourier-based OFDM [3]. The WPM can be efficiently implemented using the well-known filter bank structure introduced by [6].

The convolutional turbo coder consists of a parallel concatenation of recursive systematic convolutional RSC encoders separated by a pseudo-random interleaver[7], [8]. The main aim of RSC is to produce more high weight codes even though input contains more number of zeros [9]. One important feature of turbo codes is the iterative decoding which uses a soft-in/soft-out (SISO) like MAP (Maximum A Posteriori) decoding algorithm which was first applied to convolution codes by BahlCocke, Jelink and Raviv also known as (BCJR) algorithm [ [10],[11].

The Max-Log-MAP (MLMAP) algorithm is a good compromise between performance and complexity [17].It is very simple and, with the correction operation, also very effective [12]]. Like other methods max-log-APP algorithm calculates approximate log-likelihood ratios LLR's for each input sample as an estimate of which possible information bit was transmitted at each sample time [12], [11].

Soft in Soft Output SISO decoding of the convolutional sub codes is done with the use of a-priori information of previous decoding steps. Here only relevant formulas of the used MaxLog-APP MLMAP algorithm are given. For more details of Turbo codes and Turbo decoder, see [10],[13], [14], [15]].

The standard formulas for the MLMAP algorithm is given

$L_{\text {out }}^{e}\left(u_{k}\right)=\frac{L\left(u_{k}\right)}{2}-\left(y_{k}^{s}+L_{m}^{e}\left(u_{k}\right)\right)$

The term $L_{\text {out }}^{e}\left(u_{k}\right)$ is the information exchanged between the constituent decoders, $L\left(u_{k}\right)$ is the extrinsic information which is used as the a-priori information in the next decoder iteration, Leis an a-priori information for $u_{k}$ calculated in the previous iteration, and $\mathrm{y}^{\mathrm{s}}$ is the received channel symbol.

The additional scaling factor sused in the investigations is applied as follows:

$$
L_{\text {out }}^{e}\left(u_{k}\right)=\left[\frac{L\left(u_{k}\right)}{2}-\left(y_{k}^{s}+L_{m}^{e}\left(u_{k}\right)\right)\right] . s \quad 2
$$

\section{WAVELET PACKET BASED MCM SYSTEM MODELING DESCRIPTION}

Let the output bit stream from the spreader be $X=(x[1], x[2]$, ..., $x[N])$, is first converted from serial to parallel sequences $S_{k}$ and then modulated with $M$-array inverse wavelet packet transform (IWPT). Figures 1 shows the wavelet packet based MCM transmitter operating Mallat's fast algorithm [4].

The transmitted signal $Y$, is composed of successive $\mathrm{K}$ symbols, as the sum of $M$ amplitude modulated waveforms by $\varphi_{k}$ as:

$$
\mathrm{Y}=\sum_{\mathrm{K}}^{M-1} \mathrm{~S}_{\mathrm{K}} \emptyset_{\mathrm{K}}
$$

whereY $=(y[1], y[2], \ldots, y[n], \ldots, y[N])$, is the transmitted signal. $\mathrm{S}_{\mathrm{K}}=\left(\mathrm{s}_{\mathrm{o}}[\mathrm{k}], \mathrm{s}_{1}[\mathrm{k}], \ldots . \mathrm{S}_{\mathrm{m}}[\mathrm{k}], \ldots, \mathrm{S}_{\mathrm{N}}[\mathrm{k}]\right)$, is the output of serial to parallel converter and $\varphi_{\mathrm{k}}$,

$$
\emptyset_{k}=\left[\begin{array}{ccc}
\emptyset_{0}[1-k M] & \cdots & \emptyset_{0}[N-k M] \\
\vdots & \emptyset_{m}[n-k M] & \vdots \\
\emptyset_{M-1}[1-k M] & \cdots & \emptyset_{M-10}[N-k M]
\end{array}\right] 4
$$

is the waveforms matrix which $\varphi_{m}[n]$ represent the WPMCM basis function that are mutually Orthogonal[15] to reduce the symbol errors [16] .

In the wavelet packet scheme, we limit our analysis to subcarrier waveforms defined through a set of FIR filters, and implemented by Mallat's fast algorithm [15][16] with less complexity for wireless communication.

The Mallat algorithm implemented by quadrature mirror filter pair (QMF) that consists of the scaling filter $g$ and dilatation filter $\mathrm{h}$, and knowledge of the scaling filter and wavelet tree depth is sufficient to design the wavelet transform. The scaling

filter $\mathrm{g}$ and dilatation filter $\mathrm{h}$, and the corresponding reversed filtersg(-n) and $h(-n)$, are used to form a wavelet packet tree. These filters satisfy following conditions:

$$
\begin{aligned}
& \sum_{n=-\infty}^{\infty} g(n)=2 \\
& \sum_{n=-\infty}^{\infty} h(n) g(n-2 l)=2 \delta(l)
\end{aligned}
$$

where $\mathrm{M}$ is the length of the filters.

The carrier waveforms are obtained by iteratively filtering the signal into high and low frequency components. The waveforms $\varphi_{\mathrm{m}}[\mathrm{m}]$ are derived by $J$ successive iterations as the following recursive equations:

$$
\left\{\begin{array}{c}
\varphi_{j, 2 m}[n]=g[n] * \varphi_{\varphi_{j-1, m[n / 2]}} \\
\varphi_{j, 2 m+1}[n]=h[n] * \varphi_{\varphi_{j-1, m[n / 2]}} \\
\varphi_{0, m}[n]=\left\{\begin{array}{l}
1, n=1 \\
0, \text { else }
\end{array}\right.
\end{array}\right.
$$

Where $j$ is the iteration index, $1 \leq j \leq J$, and $m$ the waveform index $1 \leq m \leq M-1$. Y is the transmitted signal through the channel $H$, with $L$ multi-paths, $\mathrm{H}=(\mathrm{h}[0], \mathrm{h}[1], \ldots, \mathrm{h}[1], \ldots, \mathrm{h}[\mathrm{L}-$ 1]) and received signal at the output of the channel can be written as:

$R=H . Y+W$

where $\mathrm{R}=(\mathrm{r}[1], \mathrm{r}[2], \ldots, \mathrm{r}[\mathrm{n}], \ldots, \mathrm{r}[\mathrm{N}])$, is the received signal, and $\mathrm{W}=(\mathrm{w}[1], \mathrm{w}[2], \ldots, \mathrm{ws}[\mathrm{n}], \ldots, \mathrm{w}[\mathrm{N}])$ is additive white Gaussian noise (AWGN).

\section{PROPOSED SYSTEM}

At the transmitter the data stream of $i^{\text {th }}$ user, afterchannel coding with turbo encoder with rate of 0.5 . The coded data is mapped by QAM mapper then speeded by $\mathrm{i}^{\text {th }}$ spreading code. Eight pilot's carriers are added to the frame for channel compensation. The frame with pilot carriers first converted from serial to parallel sequences $S_{k}$ and then modulated with 
inverse wavelet packet transform (IWPT). Figures1 and 2, show the wavelet packet based MCM transceiver operating Mallat's fast algorithm.

The DWPT-OFDM system model with the PCCC turbo coding schemes shown in Figure 3.

This output data is converted to serial vector, and then this vector is convoluted with the channel selected in the work. The AWGN is added with specific signal to noise ratio.

At the receiver side, the serial data is converted into parallel data by serial to parallel converter. Then, the DWPT is used to find the corresponding WP domain of the parallel data. The pilot carriers is extracted and used to overcome channel distortion. Wavelet packed de-modulated data is then evaluated and converted back into the data word by demodulating the received symbol. Then, the data is de-speeded with $\mathrm{i}_{\mathrm{th}}$ spreading code QAM-demodulated then decoded to reconstruct the original data.

Haar wavelet is used in the design of the proposed model, due its simplicity and better performance. The level of transformation was 3 .

The parameters and system configuration used in the simulation are summarized as follows: -

\begin{tabular}{|l|l|}
\hline Modulation scheme & BPSK \\
\hline Number of subcarrier of FFT & 256 subcarriers \\
\hline Wavelet level & three \\
\hline Type of wavelet transform & Haar wavelet \\
\hline OFDM symbol duration & $64^{*} 10^{-6} \mathrm{sec}$ \\
\hline Required bandwidth & $5 \mathrm{MHz}$ \\
\hline Model of simulated channel & Jacks Model \\
\hline Number of path & $6 \mathrm{paths}$ \\
\hline Pilot Carriers & 8 \\
\hline Doppler frequency & $50 \mathrm{~Hz}$ \\
\hline Types of spreading code used & Walsh Hadamrd \\
& code \\
\hline Processing gain (Walsh code) & 32 \\
\hline Required BER & $10^{-4}$ \\
\hline
\end{tabular}

When the number of subcarriers is equal to 256 , the number of DWPT points is equal to 256 (128 approximation and 128 details). At the receiver, the length of data out from the DWT is equal to 256 (the first 128 subcarriers represent the received data, while the second 128 subcarriers represent the details).

\section{RESULTS}

The proposed system illustrated in Figure [3] is the wavelet Packet based MC-CDMA system. A 20 Mbps was transmitted over the system. The modulation schemes are the QAM with $\mathrm{M}=2,4,16$, and 64 . The work implied performance comparison of the proposed wavelet Packet based WPM MC-CDMA system with that of Fast Fourier Transform FFT MC-CDMA system.
A simulation of the two systems has been made using MATLAB version 2010b. The BER performance of the two systems will also be studied in different channel models which are Additive White Gaussian Noise AWGN and multipath fading channel, with a bandwidth of $5 \mathrm{MHz}$ and WalshHadamard (code 20) has been used with 32 bits.

The system uses turbo coding. First, the input binary data generated at the transmitter side is encoded with the parallel concatenated convolutional coder with both the upper and lower coder of a generator polynomial of $\left.\left[\begin{array}{llllllll}1 & 0 & 1 & 1 ; & 1 & 1 & 0 & 1\end{array}\right]\right)$ polynomial generators and a constraint length of (4). With the MAX-Log-MAP decoding algorithm which is an iterative decoding algorithm. The performance of the concatenated convolutional code system depends upon the number of iteration of the decoder.

\subsection{Performance of the Proposed System in AWGN Channel}

The channel here was modeled as an Additive White Gaussian Noise AWGN channel. Figures [6, 7,8,9 and 10] show the variations of BER versus signal to noise ratio $(\mathrm{SNR})\left(\mathrm{E}_{\mathrm{s}} / \mathrm{N}\right.$ signal power to the noise power) for AWGN channel for parallel concatenated convolutional coded data for both FFT MC-CDMA system and DWPT MC-CDMA system with QAM modulation scheme of $\mathrm{M}$ value of 2,4, 16, and 64 respectively. For $\mathrm{M}=64$ it can be noticed that BER at 10-3 was achieved at SNR of $22 \mathrm{~dB}$ for DWPT MC-CDMA system and $26 \mathrm{~dB}$ for FFT MC-CDMA system with a gain of $4 \mathrm{~dB}$ is obtained by the proposed model.

\subsection{Performance of the Proposed System in Multipath Fading Channel.}

In this section, the channel was considered to be a multipath fading channel. Simulations of parallel concatenated convolutional coded PCCC data for both FFT MC-CDMA system and DWPT MC-CDMA system were done. The performance of the mentioned systems with QAM modulation scheme of M-level of 2, 4, and16 are shown in figures [11, 12 and 13] respectively. For $M=16$ it can be noticed that BER at $10^{-3}$ was achieved at SNR of $16 \mathrm{~dB}$ for DWPT MC-CDMA system and $20.5 \mathrm{~dB}$ for FFT MC-CDMA system with gain of $4,5 \mathrm{~dB}$ was obtained by the proposed model.

\section{CONCLUSION:}

Both systems have been investigated and the results have shown that the proposed DWPT MC-CDMA system is better than the conventional system to work under different channel characteristics.

Approximately a gain of $4 \mathrm{~dB}$ or more was obtained at the AWGN channel, $4.5 \mathrm{~dB}$ gain was obtained for multipath fading channel for a $\mathrm{BER}=10^{-3}$ from the proposed MC-CDMA.

\section{REFERENCES}

[1] Xiangbin YU., Xiaodong Zhang, Dazhuan XU., Guangguo BI.," Performance Analysis of Multiband Complex Wavelet Based MC_CDMA System with Space Diversity Combining in Rayleigh Fading Channel", Wireless Personal Communication, No. 41, 2007,pp 193-206.

[2] Salih M. Salih, N Uzunglu, L. Awda Kadhim, L. A. El_Enzy, "A Proposed Model for MC_CDMA Based In_Place Wavelet Transform", 
[3] Rashmi Mishra, BaibaswataMohapatra, PERFORMANCE EVALUATION OF OFDM SYSTEM", International Journal of Engineering and Advanced Technology (IJEAT) ISSN: 2249 - 8958, Volume-1, Issue-3, February 2012.

[4] Giovanni Cherubini, EvangelkosEleftherioi, SedatOlcer, John M. Cioffi, "Filter Bank Modulation Techniques for Very High-SpeedDigital Subscriber Lines": IEEE Communications Magazine. May2000.

[5] Yuan-Pei Lin, See-May Phoong, "Perfect Discrete Multitone Modulation with Optimal Transceivers", IEEE Transactions on Signal Processing, Vol. 48 no. 6, June 2000.

[6] Mallat S.G., a wavelet tour of signal processing, 2nd ed., Academic Press, San Diego, California, 1999.

[7] Ramasmay K., Mohammed U. S., mohammed Y. A. and A. Arunagiri, " Performance comparision of convolutional and block codes",IEICE Electronic Express, V. 3, N. 13, pp, 322-327.

[8] Aqiel N. Almaamory and HusamAbduldaem.Mohammed,"Performance Evaluation and Comparison of LDPC and Turbo Coded MCCDMAJournal of Engineering, University of Baghdad, Number 4, Volume 18, April 2012

[9] Shanmugam A., A. R. Abdul Rajak, "Optimal design of forward error correcting codes for wireless communication"m, Academic open Internet journal, V. 14, 2005, http://www.acadjournal.com/.

[10] Bahl, J.L. Cocke, F. Jelinek, and J. Raviv, "Optimal decoding of linear codes for minimizing symbol error rate," IEEE Trans. Inform. Theory, vol. IT-20, pp. 284-287, Mar. 1974.
[11]HusamAbduldaem. Mohammed"PCCC MC-CDMA Combination Performance Over Multipath Rayleigh Fading Channel", Journal of Engineering, University of Baghdad, V.16, No.3 Septemper 2010.

[12] Robertson, P. Hoeher, and E. Villebrun, "Optimal and SubOptimal Maximum aPosteriori Algorithms Suitable for Turbo Decoding", IEEE Communications Theory, Vol. 8, No. 2, pp. 119-125, March-April1997.

[13]BERROU C., A. GLAVIEUX, and P. Thitimajshima: „Near Shannon limit errorcorrecting coding and decoding: Turbocodes (1)", Proc. of the IEEE ICC '93, Geneva, Switzerland, May 1993, pp. 1064-1070

[14] RobertsonP., E. Villebrun, and P. Hoeher, "A Comparison of Optimal and Sub-Optimal MAP Decoding Algorithms Operating in the Log Domain", Proceedings of the 1995 International Conference on Communications (ICC'95), Seattle, WA,USA, pp. 1009-1013, June 1995.

[15]HagenauerJ., P. Robertson and L. PapkeE: "Iterative (turbo) decoding ofsystematic convolutional codes with the MAP and SOVA algorithms", in Proc. of theITG, Munich, Oct. 1994

[16] HalehHosseini, NorsheilaFisal, \&Sharifah K. Syed-Yusof, "Wavelet Packet based Multicarrier Modulation for Cognitive UWB Systems", Signal Processing - An International Journal (SPIJ), Volume (4): Issue (2) 75.

[17]Vogt J., K. KOORA, A. FINGER and G. FETTWEIS: "Comparison of DifferentTurbo Decoder Realizations for IMT-2000", in Proc. of the IEEE GLOBECOM '99, Riode Janeiro, Dec. 1999, pp. 2704-2708.

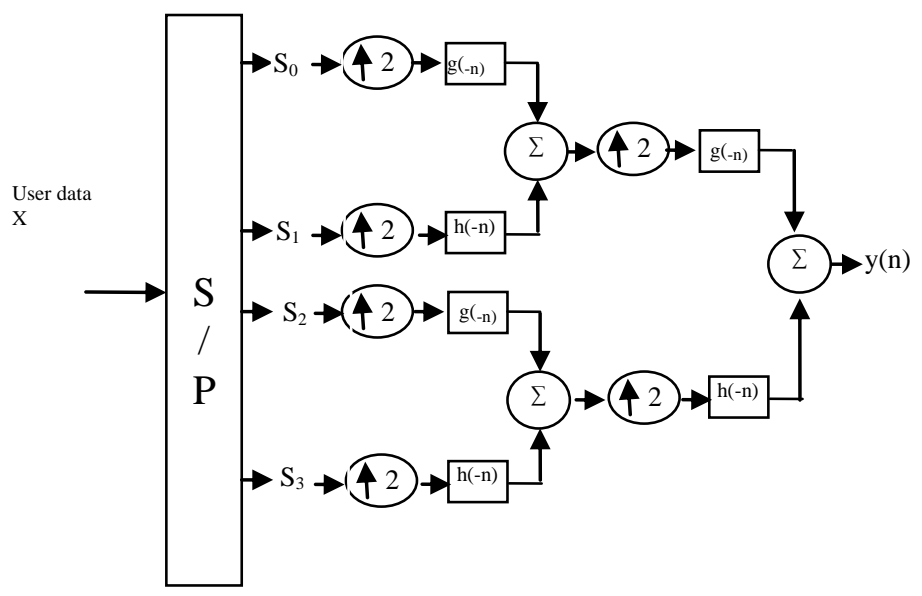

Level 2 decomposition

Level 1 decomposition

Fig 1: Two level inverse wavelet packet transform using Mallat Algorithm 


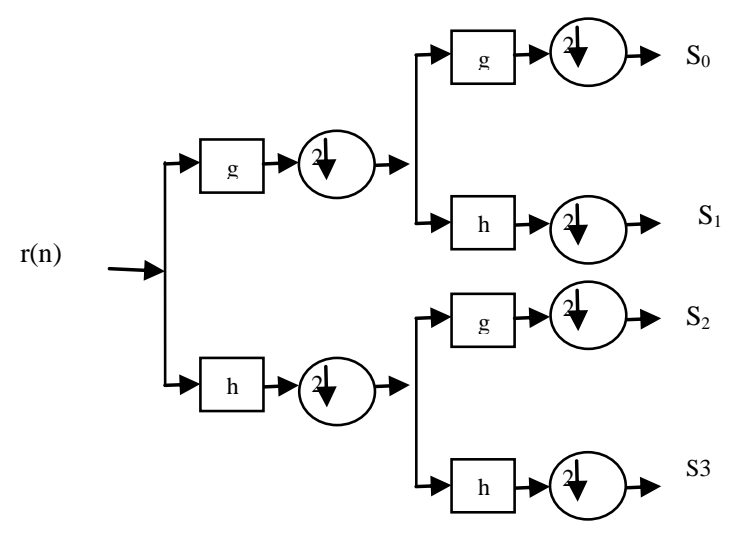

Fig 2: Two level wavelet packet transform using Mallat Algorithm

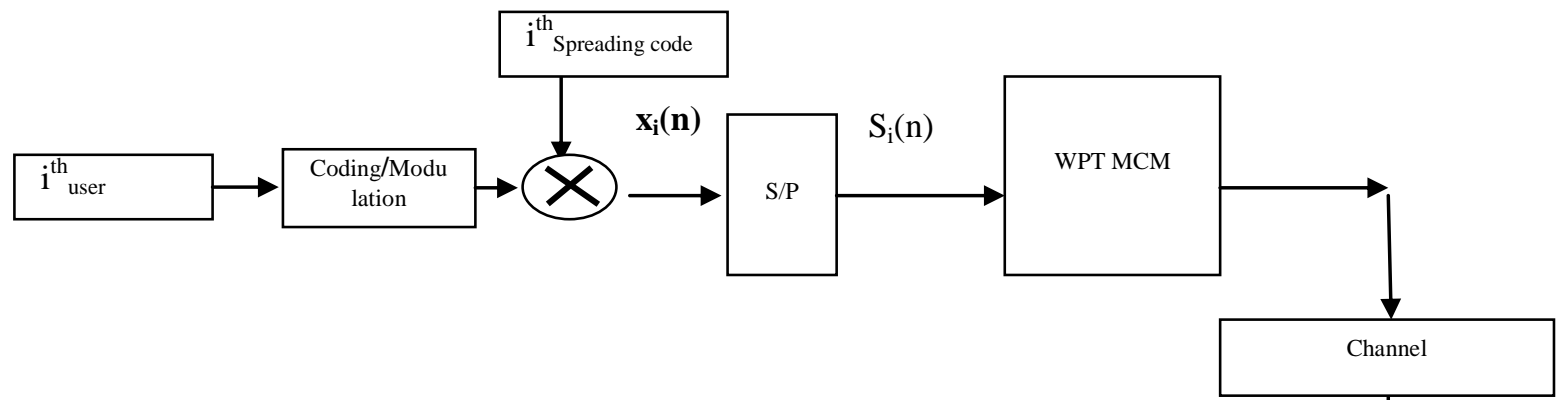

(a) Transmitter

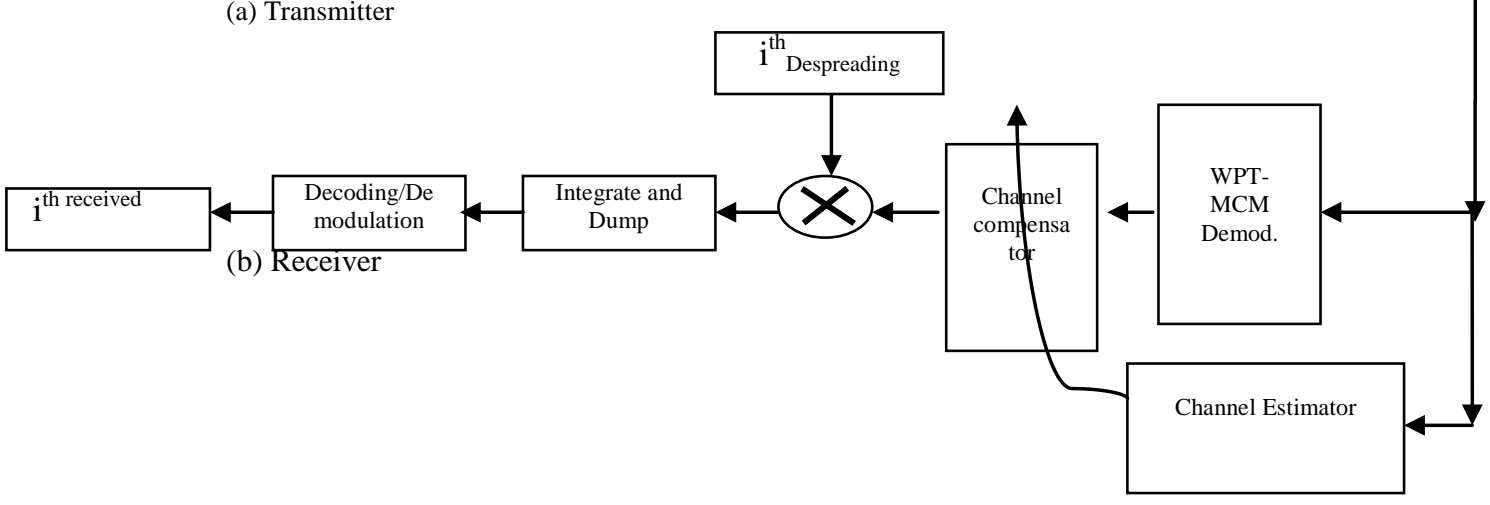

Figure 3: Block diagram of a transceiver via WPMCM 


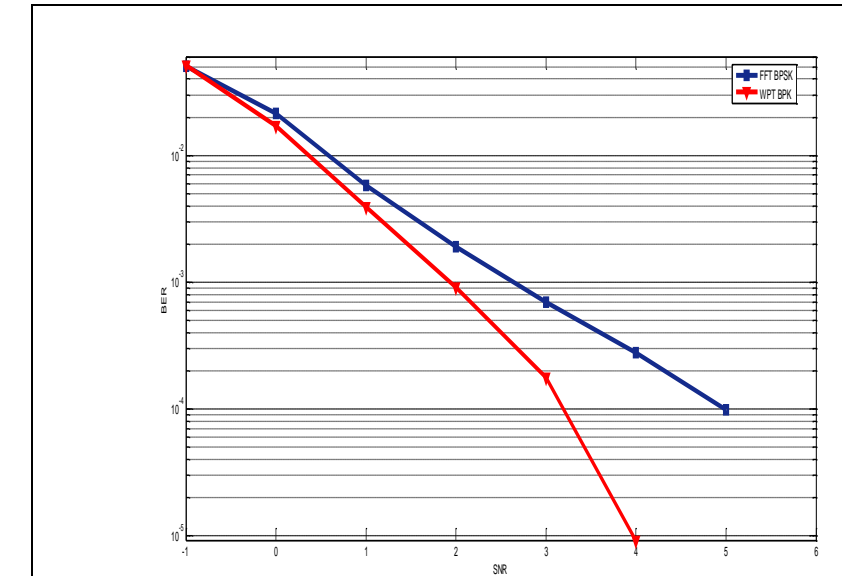

Figure (4) PCCC coded MC-CDMA performance for AWGN channel with BPSK modulation.

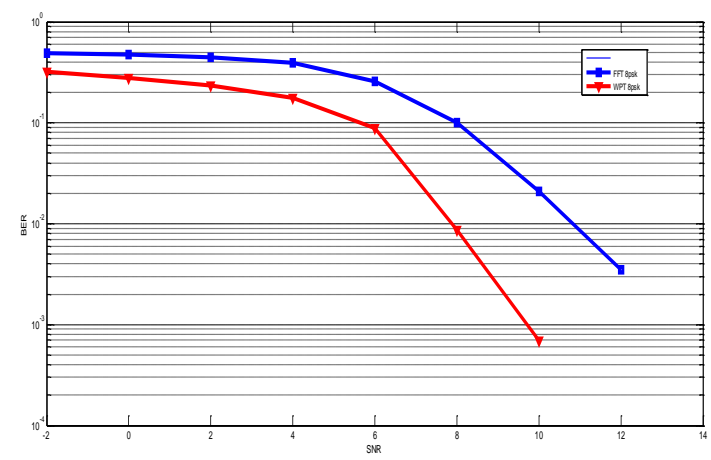

Figure (6) PCCC coded MC-CDMA performance for AWGN channel with 8PSK modulation.

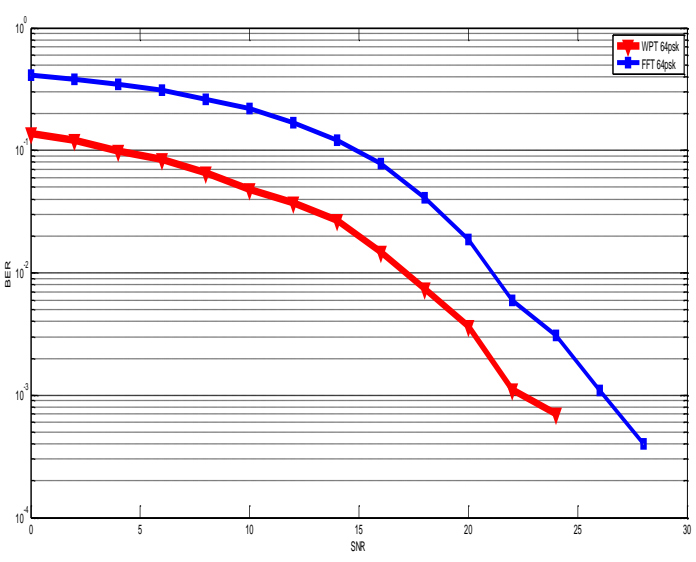

Figure (8) PCCC coded MC-CDMA performance for AWGN channel with 64PSK modulation.

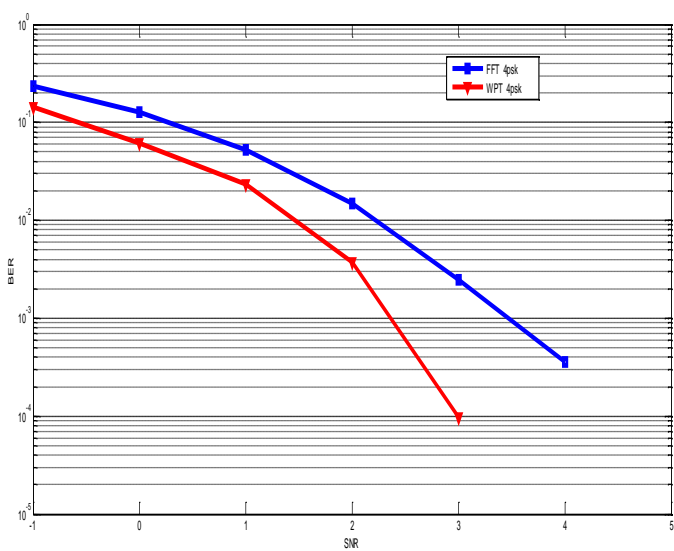

Figure (5) PCCC coded MC-CDMA performance for AWGN channel with 4PSK modulation.

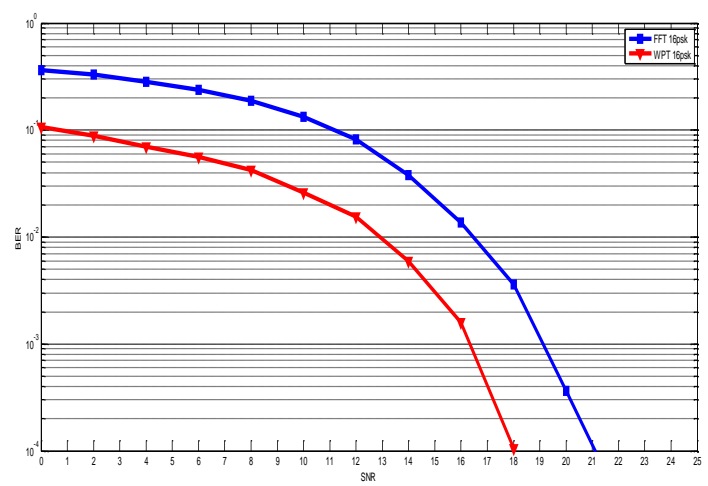

Figure (7) PCCC coded MC-CDMA performance for AWGN channel with 16PSK modulation.

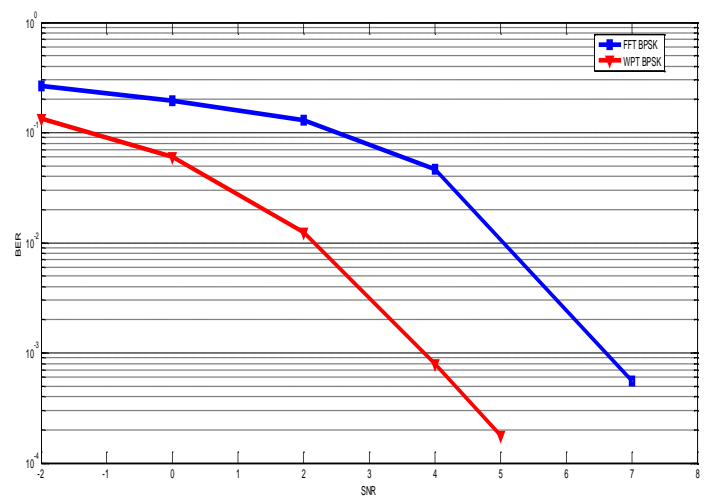

Figure (9) PCCC coded MC-CDMA performance for multipath fading channel with BPSK modulation. 


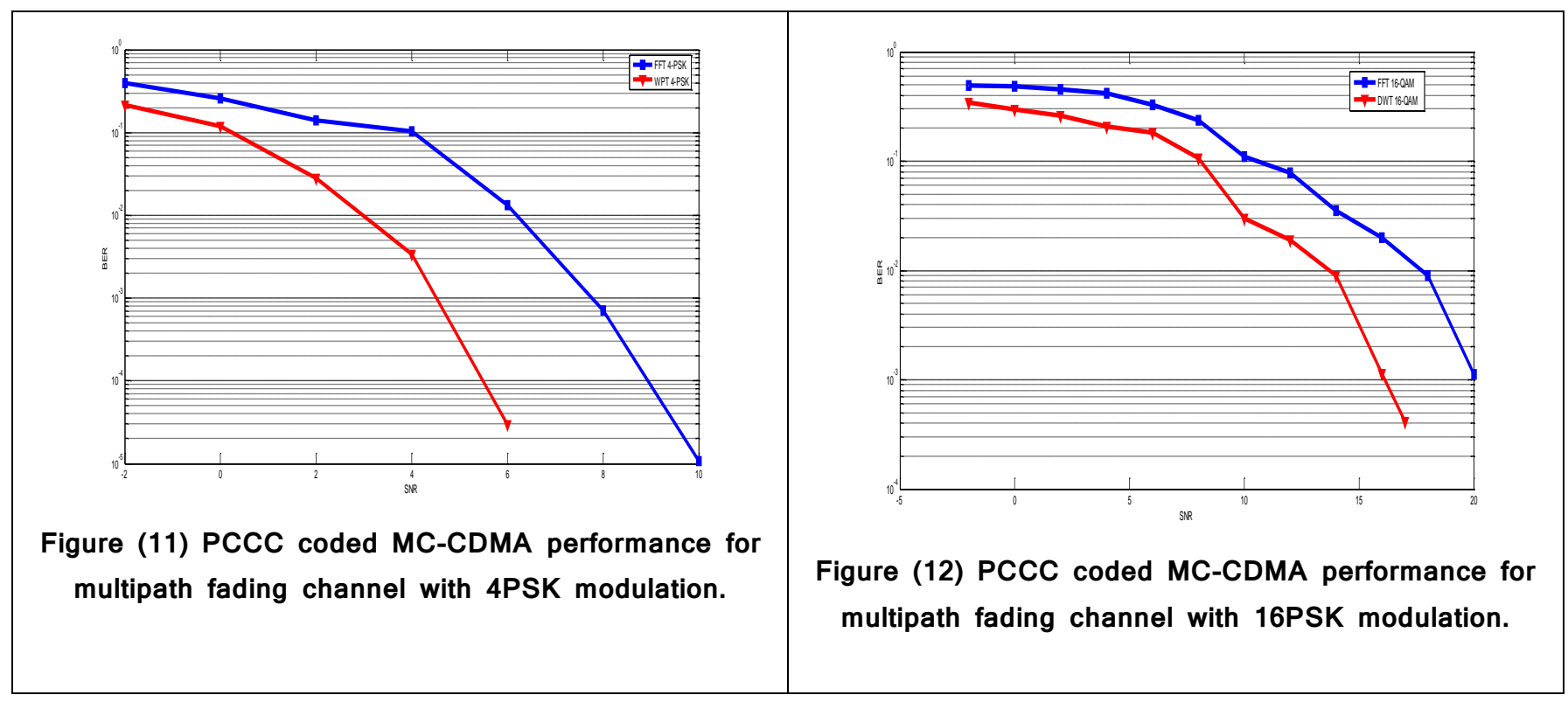

